Clinical trial

\title{
Clinical and radiologic assessments to predict breast cancer pathologic complete response to neoadjuvant chemotherapy
}

\author{
Anne F. Schott ${ }^{1}$, Marilyn A. Roubidoux ${ }^{5}$, Mark A. Helvie ${ }^{5}$, Daniel F. Hayes ${ }^{1}$, Celina G. Kleer ${ }^{2}$, \\ Lisa A. Newman ${ }^{3}$, Lori J. Pierce ${ }^{4}$, Kent A. Griffith ${ }^{6}$, Susan Murray ${ }^{7}$, Karen A. Hunt ${ }^{8}$, Chintana \\ Paramagul $^{5}$, and Laurence H. Baker ${ }^{1}$ \\ ${ }^{1}$ Hematology/Oncology Division, Department of Internal Medicine, University of Michigan, USA; ${ }^{2}$ Department of \\ Pathology, University of Michigan, USA; ${ }^{3}$ Department of Surgery, University of Michigan, USA; ${ }^{4}$ Department of \\ Radiation Oncology, University of Michigan, USA; ${ }^{5}$ Department of Radiology, University of Michigan, USA; \\ ${ }^{6}$ Comprehensive Cancer Center, University of Michigan, USA; ${ }^{7}$ School of Public Health, University of Michigan, \\ USA $;{ }^{8}$ Henry Ford Hospital, USA
}

Key words: breast cancer, docetaxel, doxorubicin, magnetic resonance imaging, mammography, neoadjuvant chemotherapy, pathologic complete response, response prediction, ultrasound

\section{Summary}

Purpose. To prospectively compare the ability of clinical examination, mammography, vascularity-sensitive ultrasound, and magnetic resonance imaging (MRI) to determine pathologic complete response (CR) in breast cancer patients undergoing neoadjuvant chemotherapy.

Patients and methods. Participants were women with primary measurable, operable invasive breast cancer (Stages I-III) who presented to the University of Michigan Breast Care Center. Eligibility criteria were based on clinical need for chemotherapy as part of the overall treatment plan. The chemotherapy consisted of doxorubicin and docetaxel administered every 3 weeks for four cycles. Tumor size measurements by physical examination and by the three imaging modalities were performed before chemotherapy was initiated and after its completion, prior to definitive surgery. Response criteria were pre-specified in this prospective design, and study radiologists analyzed the mammographic, sonographic and MRI image sets blinded to information from the other modalities and blinded to final histological diagnosis. The pathologic CR rate obtained by the clinical and imaging modalities was compared to pathologic $\mathrm{CR}$ as determined pathologically.

Results. 41 of 43 enrolled patients had a determination of pathologic response, and 4 patients had a pathologic CR to this chemotherapy $(9.8 \%)$. The accuracy of physical examination, mammography, ultrasound, and MRI in determining pathologic CR was $75,89,82$, and $89 \%$ respectively (NS).

Conclusion. Biopsy after neoadjuvant chemotherapy remains absolutely necessary to determine pathologic $\mathrm{CR}$ to neoadjuvant chemotherapy, as the accuracy of current imaging modalities is insufficient to make this determination. The accuracy of mammography, vascularity-sensitive ultrasound, and MRI were not observed to be significantly different.

\section{Introduction}

Adjuvant systemic therapy reduces mortality in patients with newly diagnosed breast cancer. Recent clinical trials have shown that preoperative, or neoadjuvant, chemotherapy is equally effective as adjuvant chemotherapy and can result in a higher incidence of breast preservation $[1,2]$. However, selection of candidates for breast conservative therapy after neoadjuvant chemotherapy is not always straightforward. Physical examination may suggest a complete response to therapy, while mammography shows extensive residual calcifications and sometimes even increased calcifications [3, 4]. Development of sensitive and specific clinical imaging methods that can determine whether there is residual disease could spare patients who had a complete pathologic response from having further surgery. For those patients with residual disease, accurate delineation of residual cancer could simplify the selection of candidates for breast conservation therapy, thereby reducing the number of lumpectomy procedures with positive margins by identifying those patients who still have extensive disease following chemotherapy [5].

In addition to enhanced breast preservation rates, neoadjuvant chemotherapy permits observation of chemoresponsiveness of the tumor. Patients with tumors that are highly chemotherapy sensitive, as measured by the ability of a chemotherapy regimen to induce 
pathologic complete response (CR), have improved long-term disease free survival [6-9]. Ideally, a patient's response to neoadjuvant chemotherapy could be used to individually tailor systemic treatment, although the clinical benefit of this approach has not yet been proven. Non-invasive imaging methods that can accurately determine pathologic CR after a short course of chemotherapy would be of great value in the execution of clinical trials that test the utility of assigning different treatment type or duration based on response to neoadjuvant chemotherapy.

Common imaging modalities used in the evaluation of patients with primary breast cancer include mammography, ultrasound, and magnetic resonance imaging (MRI). There is extensive literature on the use of these three modalities in measuring breast primary tumors and the extent of residual disease after chemotherapy [4, 10-27]. However, we found no study to prospectively evaluate the ability of imaging to predict pathologic CR following neoadjuvant chemotherapy. In this prospectively designed study, the primary goal was to compare the ability of MRI, vascularity-sensitive ultrasound, mammography, and clinical examination to determine complete pathologic response.

\section{Patients and methods}

\section{Entry criteria}

Eligible patients were women with primary measurable, operable invasive breast cancer (clinical T1, 2, 3; N0, 1, 2; M0), based on tumor-nodes-metastases (TNM) Criteria as detailed in Handbook for Staging of Cancer, American Joint Committee on Cancer, 5th Edition, and confirmed by core needle biopsy. Tumor size was required to be $\geq 1 \mathrm{~cm}$. Immunohistochemical staining for estrogen receptor, progesterone receptor, and Her-2 neu were obtained on the initial diagnostic sample. Patients had an ECOG Performance status of $0-2$, and were in good general health with normal complete blood count and comprehensive metabolic profile. Patient characteristics are outlined in Table 1.

Patients were excluded if their breast tumors were not measurable by any of the modalities, including mammography, ultrasound, and MRI, or if the tumor was diagnosed by incisional biopsy that did not leave measurable disease by at least one modality. Patients could not have received any prior chemotherapy, radiation therapy, or tamoxifen therapy for their current breast cancer.

All patients signed an informed consent regarding the experimental nature of this therapy after approval by the University of Michigan Institutional Review Board.

\section{Treatment plan}

\section{Diagnosis and staging}

Patients presented for evaluation at the Breast Care Center at the University of Michigan. The plan for
Table 1. Characteristics of patients at baseline

\begin{tabular}{|c|c|c|}
\hline & $n=43$ & $\%$ \\
\hline \multicolumn{3}{|l|}{ Age, years } \\
\hline Median & 48 & \\
\hline Range & $26-66$ & \\
\hline \multicolumn{3}{|c|}{ Size of tumor (physical examination) } \\
\hline$\leq 2.0 \mathrm{~cm}$ & 6 & 14 \\
\hline $2.1-5.0 \mathrm{~cm}$ & 23 & 53 \\
\hline$\geq 5.1 \mathrm{~cm}$ & 13 & 30 \\
\hline Not assessable & 1 & 2 \\
\hline \multicolumn{3}{|l|}{$\begin{array}{l}\text { Pre-chemotherapy lymph node } \\
\text { status (includes SLNB) }\end{array}$} \\
\hline Negative & 14 & 33 \\
\hline Positive & 29 & 67 \\
\hline \multicolumn{3}{|c|}{$\begin{array}{l}\text { Pre-chemotherapy clinical/pathologic } \\
\text { stage (includes SLNB) }\end{array}$} \\
\hline I & 3 & 7 \\
\hline II & 34 & 79 \\
\hline III & 6 & 14 \\
\hline \multicolumn{3}{|l|}{ Estrogen receptor status } \\
\hline Positive & 25 & 58 \\
\hline Negative & 18 & 42 \\
\hline \multicolumn{3}{|c|}{$\begin{array}{l}\text { Histology (on pre-chemotherapy } \\
\text { core biopsy) }\end{array}$} \\
\hline Invasive ductal carcinoma & 29 & 67 \\
\hline Invasive lobular carcinoma & 3 & 7 \\
\hline Anaplastic & 6 & 14 \\
\hline Mixed ductal and lobular & 2 & 5 \\
\hline Not otherwise specified & 3 & 7 \\
\hline
\end{tabular}

neoadjuvant chemotherapy was approved by the Breast Care Center's multidisciplinary Tumor Board. All patients had primary breast tumor measurements by palpation performed by their physician at the time of initial presentation.

In patients felt to be potential candidates for conservative therapy after neoadjuvant treatment, either a titanium clip was placed in the tumor, or a small tattoo was placed over the palpable mass to guide later excision.

\section{Procedures for response evaluation}

Physical examination and tumor size measurements were performed on day one of each cycle of chemotherapy and at the end of chemotherapy before surgery. The three imaging modalities (mammography, vascularity-sensitive ultrasound, and MRI) were performed at baseline (before chemotherapy was initiated) and at the end of the fourth cycle of chemotherapy, before definitive surgery. Radiologists analyzed the mammographic, sonographic and MRI image sets blinded to information about these cases from the other modalities and additionally blinded to final histological information at excision. The response criteria as defined prospectively for each modality are presented in Table 2 . 


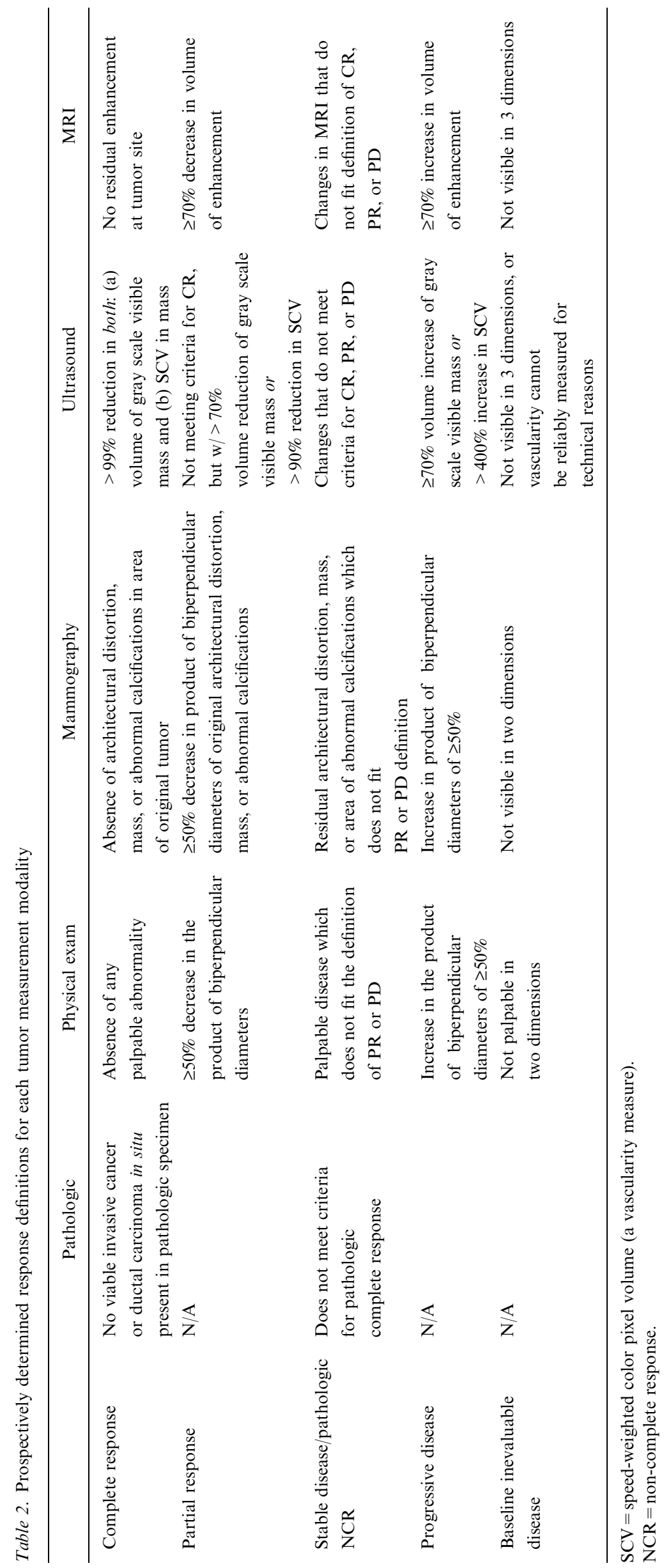




\section{Mammography}

Each patient received diagnostic mammography, which included two mammographic views of the involved breast (craniocaudal, mediolateral oblique) and additional views (mediolateral, spot magnification views) as clinically indicated. Measurement in three dimensions was performed manually by an experienced FDA certified radiologist. The mammographic views showing the best delineation of tumor margins were used for subsequent measurements. For masses, perimeter margins were used. For calcifications, the greatest extent is measured in each projection. These cases were analyzed by a single reader (MAH).

\section{$M R I$}

MRI examinations were performed on a 1.5 Tesla General Electric (GE) magnet, using a Medrad dualphased array dedicated breast coil for optimal resolution and signal to noise ratio. Three dimensional volume acquisition gradient echo sequences were performed with fat saturation before and after the intravenous administration of $0.15 \mathrm{mmol} / \mathrm{kg}$ gadolinium. A hybrid sequence was used of rapid dynamic imaging combined with high resolution imaging. Dynamic imaging was performed using $30 \mathrm{~s}$ intervals for $8 \mathrm{~min}$, in addition to an intervening high resolution sequence performed at $4.5 \mathrm{~min}$. Using this method, signal enhancement curves were generated at a workstation for areas of known tumor, and also for additional unsuspected areas of suspicious abnormal early rapid vascular enhancement. Thus, previously unsuspected multifocal disease, based on physical examination and mammography, were also assessed for response to chemotherapy. Tumor volume was measured from 3D angiogram data constructed from subtraction data of dynamic early enhancement images minus the immediately preceding non-contrast images. Occasionally, on post chemotherapy studies, 3D angiogram was constructed from later dynamic images due to 'slowing' of uptake of contrast in response to chemotherapy in a lesion with persistent suspicious morphology. Subjective tumor morphology was characterized using descriptors defined by Abraham et al. [28]. Non-enhancing masses were considered non-suspicious.

To determine tumor response to chemotherapy, the respective post-therapy MRI tumor enhancement volumes were compared to the pre-therapy breast MRI tumor enhancement volume by a single reader (KAH and/or CP). Residual tumor enhancement volume may have been less than the total residual tumor volume as determined by $\mathrm{T} 1$ and $\mathrm{T} 2$ weighted imaging. Residual volume of enhancement, hypothesized to represent residual metabolically active disease, was distinguished from the larger volume seen on non-contrast imaging composed of tumor and post treatment tumor fibrosis.

\section{Ultrasound}

Vascularity-sensitive ultrasound was performed in each patient. In each study, the mass was localized and imaged in three dimensions (3D) with a GE Logiq 700 scanner. The skin over the breast mass was coated with a warmed, non-staining ultrasound scanning gel and the breast was stabilized by stretching a two mil polyethylene film, coated with ultrasonic coupling gel, across the chest. The film was coated externally with gel and the breast was scanned in 3D by a combination of high resolution gray scale and color flow imaging. The compact hand-driven scanner depicted in [29] was employed along with the $739 \mathrm{~L}$ (7.5 MHz, $40 \mathrm{~mm}$ linear) scanhead. For large, highly attenuating lesions, the lower-frequency, wide field of view GE M7, 3-7 MHz, slightly curved matrix array was used. Both whole-volume gray scale and color Doppler image sets were acquired using the methods described by Bhatti et al. [30]. The same procedure was performed after four cycles of chemotherapy. The radiologist (MAR) selected the mass in each image volume and the volume and vascularity of the masses were computed using the method described previously [30].

\section{Chemotherapy regimen}

The chemotherapy consisted of doxorubicin $50 \mathrm{mg} / \mathrm{m}^{2}$ intravenously (IV), and docetaxel $75 \mathrm{mg} / \mathrm{m}^{2}$ IV, 1530 min after administration of doxorubicin and infused over $1 \mathrm{~h}$, on day one every 3 weeks for four cycles. Patients received premedication with dexamethasone, $8 \mathrm{mg}$ twice daily for 3 days, beginning the day prior to chemotherapy. Antiemetics included a serotonin antagonist, dexamethasone, and a phenothiazine derivative. Either filagrastim or pegfilagrastim were used prophylactically after experience with the first 15 patients suggested a high rate of neutropenic fever. Patients had repeat physical examination by the same physician on day one of each cycle of chemotherapy. Chemotherapy was delivered for the full four cycles as long as there was no undue toxicity, and there was no evidence of disease progression on physical examination.

\section{Post-chemotherapy surgical management}

Each case was discussed again at the Breast Care Center multidisciplinary Tumor Board, and final recommendations for mastectomy versus breast preservation were made. Results of the imaging studies were available to the clinicians and were used to make the surgical decision.

All patients who underwent breast preservation had to achieve negative surgical margins or else undergo mastectomy. Patients who chose breast preservation also received radiation therapy (RT) to the breast. Among patients who presented with microcalcifications, a post-biopsy mammogram showing no residual microcalcifications was required before proceeding to RT to the breast. Patients with clinically positive nodes prior to chemotherapy, or with a positive node at time of pretreatment sentinel node biopsy, had a Level I and II axillary node dissection at the completion of chemotherapy and received regional nodal radiation after surgery and chemotherapy were completed. 


\section{Statistical analysis}

This trial was designed to compare the ability of mammography, vascularity-sensitive ultrasound, MRI, and physical examination to predict pathologic CR. In the original study design, it was assumed that mammography had a $78.6 \%$ probability of correctly identifying pathologically determined complete responders or pathologically determined non-responders [3]. In order to have $90 \%$ power to detect an improvement of $15 \%$ beyond the $78.6 \%$ anticipated success rate using mammography, the study was to accrue 104 patients.

An unplanned review of the data conducted after 43 patients were evaluated suggested that the proportion of patients achieving a complete pathological response was low (approximately 10\%); and that the agreement between the diagnosis of response from the tumor measuring modalities and the confirmed pathological responding patients was so low that the original study objective as stated could not be reached regardless of sample size. Therefore, the study was closed to further accrual. This manuscript describes the treatment experience of these 43 patients, the response rates, and the experience with the use of multiple imaging modalities in identifying complete pathologic response.

McNemar's test of dependent proportions was used to compare response diagnoses between physical examination and the imaging modalities with pathologically determined response. Responses were dichotomized to complete response versus non-complete response for each modality. Exact $p$-values for the test statistics were calculated and considered significant if they were at or below $5 \%$.

\section{Results}

\section{Chemotherapy delivery}

Between November 2000 and December 2002, 43 patients were enrolled on this study. All but 1 of the 43 patients received all four cycles of chemotherapy (cycle delivery $=99.1 \%$ ). The patient who did not receive the fourth cycle was suspected to have progressed on physical examination, but on subsequent evaluation of the surgical specimen a liquid-filled tumor was found.

\section{Response by imaging modalities}

The prospectively determined response definitions are summarized in Table 2. The overall clinical response rates $(\mathrm{CR}+\mathrm{PR})$ as measured by each imaging modality and physical examination were very similar, at 74 $78 \%$ (Table 3). Overall, there were no significant differences in the proportions of the diagnosed response categories by the imaging modality when compared to pathologically determined response, although statistical power was an issue in detecting differences. In evaluable patients, physical examination was more likely to indicate a complete clinical response than were any of the imaging studies In fact, in 36 patients who completed both examinations, physical examination was significantly more likely to diagnose complete response $(30.6 \%)$ than was MRI $(5.6 \%)(p=0.004)$. However, in common patients the diagnoses by physical examination were observed to be similar to those for mammography $(n=39, p=0.11)$ and ultrasound $(n=40, p=0.07)$.

\section{Pathologic complete response}

The study was originally designed to compare the ability of mammogram, vascularity-sensitive ultrasound, MRI, and physical examination to distinguish between pathologic CR and non-CR of the breast tumors. Forty-one of forty-three evaluable patients had a determination of pathologic response, and four patients had a pathologic CR in the breast to this chemotherapy (9.8\%). This rate is nearly identical to rates in previously reported studies using a similar regimen $[31,32]$. There was only one case in which all four modalities predicted pathologic CR and pathologic $\mathrm{CR}$ was present. In three other cases of pathologic CR, extensive fibrosis or biopsy site changes were present on final pathological review, which may have affected the specificity of the radiologic findings.

The accuracy of each modality in distinguishing pathologic CR versus non-CR for those patients who had measurable disease by the modality is reported in Table 4 . The sensitivity of physical examination, mammography, ultrasound, and MRI for detecting a pathologic CR was 50, 50, 25, and $25 \%$, respectively. The specificity (those patients without a pathologic CR for whom the modality suggested residual disease) was 78 , 94,89 , and $97 \%$, respectively. The overall accuracy of physical examination, mammography, ultrasound, and MRI in predicting pathologic CR was $75,89,82$, and $89 \%$. In this regard, the positive predictive values (that a negative study predicted for pathologic CR) for physical examination, mammography, ultrasound, and MRI were $2 / 10(20 \%), 2 / 4(50 \%), 1 / 5(20 \%)$, and $1 / 2(50 \%)$. The negative predictive values (that a positive study predicted for pathologic NCR) were 93, 94, 91, and $94 \%$, respectively.

\section{Discussion}

In this study we explored the ability of clinical modalities to predict pathologic complete response in the breast, because accurate prediction of pathologic CR, as opposed to a clinical CR or PR, may (1) define a subset of patients who do not need a surgical procedure after neoadjuvant chemotherapy or (2) define a subset of patients with a worse/better prognosis who would/ would not benefit from additional systemic chemotherapy. However, even with state-of-the-art breast MRI and vascularity-sensitive ultrasound, we were not able to accurately predict pathologic CR. In over half of the 
Table 3. Breast tumor responses as measured by each modality

\begin{tabular}{llllll}
\hline & Pathology & Physical exam & Mammography & Ultrasound & MRI \\
\hline Complete response & $4 / 41(9.8 \%)$ & $11 / 41(27 \%)$ & $4 / 39(10 \%)$ & $5 / 40(13 \%)$ & $2 / 36(6 \%)$ \\
Partial response & NA & $20 / 41(49 \%)$ & $25 / 39(64 \%)$ & $25 / 40(63 \%)$ & $26 / 36(72 \%)$ \\
Stable disease/Pathologic NCR & $37 / 41(90.2 \%)$ & $10 / 41(24 \%)$ & $8 / 39(20 \%)$ & $8 / 40(20 \%)$ & $7 / 36(19 \%)$ \\
Progressive disease & NA & $0 / 41(0 \%)$ & $2 / 39(5 \%)$ & $2 / 40(5 \%)$ & $1 / 36(3 \%)$ \\
Response rate (CR + PR) & NA & $76 \%$ & $74 \%$ & $76 \%$ & $78 \%$ \\
Study performed and evaluable & $41 / 43(95 \%)$ & $41 / 43(95 \%)$ & $39 / 43(91 \%)$ & $40 / 43(93 \%)$ & $36 / 43(84 \%)$ \\
Not evaluable by this modality & $2 / 43^{\mathrm{a}}(5 \%)$ & $1 / 43(2 \%)$ & $4 / 43(9 \%)$ & $3 / 43(7 \%)$ & $1 / 43(2 \%)$ \\
Study not performed & $0 / 43(0 \%)$ & $1 / 43(2 \%)$ & $0 / 43(0 \%)$ & $0 / 43(0 \%)$ & $6 / 43(14 \%)$ \\
\hline
\end{tabular}

${ }^{a}$ One patient received 2 additional cycles of chemotherapy, one patient after review could not be confirmed to have a breast mass separate from the lymph nodes.

cases where pathologic $\mathrm{CR}$ was achieved, the imaging modalities still suggested residual cancer.

Prior studies evaluating the use of mammography, ultrasound, MRI, and clinical examination in neoadjuvant chemotherapy have shown that imaging modalities, particularly MRI, can predict the size of residual disease following neoadjuvant chemotherapy $[5,13,26,27,33]$. Other studies have shown that use of imaging early on in treatment can predict response to the prescribed therapy $[22,24]$. These studies were based on the premise that such predictions would lead to early changes in therapy and would benefit patients. However, the responses predicted by imaging in these studies were not pathologic CRs, and responses short of pathologic CR are not surrogates for meaningful clinical benefit such as disease free or overall survival. It is doubtful that use of imaging technology to predict partial responses will lead to improvement in patient outcomes.

Several previous papers have suggested that MRI is the most accurate imaging modality in measuring response to chemotherapy. In general, these studies were looking at the absolute measurement of residual disease and not the attainment of pathologic CR. The 'superiority' of MRI in such studies is expected due to its high sensitivity and low specificity for residual disease in a patient population unlikely to achieve pathologic CR. It is likely that MRI will be less accurate in a dataset with a higher pathologic $\mathrm{CR}$ rate, and so it may be worthwhile to re-examine the relative accuracy of these imaging methods for detection of pathologic $\mathrm{CR}$ within a patient population more likely to achieve pathologic CR. For example, it has been shown that more prolonged chemotherapy regimens of anthracyclines and taxanes have pathologic CR rates of 25-30\% [34], and a recent study using trastuzumab in the neoadjuvant setting for Her-2 neu positive patients reported a complete pathologic response rate of $67 \%$ [35] (Abstract). Additionally, advances in gene expression profiling may make it possible to select for study those patients with a higher likelihood of complete pathologic response [36, 37].

These imaging modalities performed best in negative prediction (identifying when pathologic $\mathrm{CR}$ was not achieved). Assuming that (1) pathologic CR is a desired goal prior to surgery in order to optimize patient outcomes, and (2) longer durations of therapy may be more effective in producing pathologic $\mathrm{CR}$, one potential use of breast imaging in neoadjuvant chemotherapy may be to determine those patients who did not have a pathologic CR after induction chemotherapy, and to offer these patients more therapy prior to definitive surgery. Recently completed multi-institutional trials of neoadjuvant chemotherapy, such as the National Surgical Adjuvant Breast Project's trial NSABP B-27 [34], will help to establish whether patients who do not achieve pathologic $\mathrm{CR}$ to four cycles of chemotherapy will benefit from additional chemotherapy. However, given the significant false-positive rate of imaging in our study, such a use should be combined with image-directed biopsy to confirm the continued presence of viable tumor.

We conclude that biopsy after neoadjuvant chemotherapy remains absolutely necessary to determine pathologic CR to short courses of neoadjuvant

Table 4. Concordance between the pathologically determined response and the diagnosis by tumor measurement modality

\begin{tabular}{|c|c|c|c|c|}
\hline \multirow[t]{2}{*}{ Pathologic response } & \multicolumn{4}{|l|}{ Modality, $n(\%)$} \\
\hline & Physical exam & Mammography & Ultrasound & MRI \\
\hline $\mathrm{CR}$ & $2 / 4(50 \%)$ & $2 / 4(50 \%)$ & $1 / 4(25 \%)$ & $1 / 4(25 \%)$ \\
\hline NCR & $28 / 36(78 \%)$ & $32 / 34(94 \%)$ & $31 / 35(89 \%)$ & $30 / 31(97 \%)$ \\
\hline Overall agreement & $30 / 40(75 \%)$ & $34 / 38(89 \%)$ & $32 / 39(82 \%)$ & $31 / 35(89 \%)$ \\
\hline Positive predictive value for pathologic CR & $2 / 10(20 \%)$ & $2 / 4(50 \%)$ & $1 / 5(20 \%)$ & $1 / 2(50 \%)$ \\
\hline Negative predictive value for pathologic CR & $28 / 30(93 \%)$ & $31 / 34(94 \%)$ & $31 / 34(91 \%)$ & $31 / 33(94 \%)$ \\
\hline
\end{tabular}

$\mathrm{CR}=$ complete response $. \mathrm{NCR}=$ non-complete response. 
chemotherapy, as the accuracy of current imaging modalities is insufficient to make this determination. It is possible that imaging modalities could play a role in identifying patients without a pathologic $\mathrm{CR}$, who may benefit from a longer and/or modified neoadjuvant treatment regimen, in combination with imaging-directed core biopsy. This role of imaging should be tested prospectively in a patient population treated with a regimen that is expected to result in a higher pathologic $\mathrm{CR}$ rate.

\section{Acknowledgements}

This research was supported (in part) by the National Institutes of Health through the University of Michigan's Cancer Center Support Grant (5 P30 CA46592). Additional support was provided by Aventis Pharmaceuticals, the General Clinical Research Center Grant M01 RR000042, and the Radiological Society of North America Research and Education Fund.

\section{References}

1. Fisher B, Brown A, Mamounas E, Wieand S, Robidoux A Margolese R, Cruz A Jr, Fisher E, Wickerham D, Wolmark N, DeCillis A, Hoehn J, Lees A, Dimitrov N: Effect of preoperative chemotherapy on local-regional disease in women with operable breast cancer: findings from National Surgical Adjuvant Breast and Bowel Project B-18. J Clin Oncol 15: 2483-2493, 1997

2. Newman LA, Buzdar AU, Singletary SE, Kuerer HM, Buchholz T, Ames FC, Ross MI, Hunt KK: A prospective trial of preoperative chemotherapy in resectable breast cancer: predictors of breast-conservation therapy feasibility. Ann Surg Oncol 9: 228234, 2002

3. Helvie M, Joynt L, Cody R, Pierce L, Adler D, Merajver S: Locally advanced breast carcinoma: accuracy of mammography versus clinical examination in the prediction of residual disease after chemotherapy. Radiology 198: 327-332, 1996 Feb

4. Vinnicombe SJ, MacVicar AD, Guy RL, Sloane JP, Powles TJ, Knee G, Husband JE: Primary breast cancer: mammographic changes after neoadjuvant chemotherapy, with pathologic correlation. Radiology 198: 333-340, 1996

5. Mumtaz H, Davidson T, Spittle M, Tobias J, Hall-Craggs MA, Cowley G, Taylor I: Breast surgery after neoadjuvant treatment. Is it necessary?. Eur J Surg Oncol 22: 335-341, 1996

6. Sataloff D, Mason B, Prestipino A, Seinige U, Lieber C, Baloch Z: Pathologic response to induction chemotherapy in locally advanced carcinoma of the breast: a determinant of outcome. J Am Coll Surg 180: 297-306, 1995

7. Robertson J, Ellis I, Pearson D, Elston C, Nicholson R, Blamey R: Biological factors of prognostic significance in locally advanced breast cancer. Breast Cancer Res Treat 29: 259-264, 1994

8. Chang J, Powles T, Allred D, Ashelty S, Clark G, Makris A, Assersohn L, Gregory R, Osborne C, Dowsett M: Biologic markers as predictors of clinical outcome from systemic therapy for primary operable breast cancer. J Clin Oncol 17: 3058-3063, 1999

9. Kuerer HM, Newman LA, Smith TL, Ames FC, Hunt KK, Dhingra K, Theriault RL, Singh G, Binkley SM, Sneige N, Buchholz TA, Ross MI, McNeese MD, Buzdar AU, Hortobagyi GN, Singletary SE: Clinical course of breast cancer patients with complete pathologic primary tumor and axillary lymph node response to doxorubicin-based neoadjuvant chemotherapy [comment]. J Clin Oncol 17: 460-469, 1999

10. Fornage BD, Toubas O, Morel M: Clinical, mammographic, and sonographic determination of preoperative breast cancer size. Cancer 60: 765-771, 1987

11. Flanagan FL, McDermott MB, Barton PT, Pilgram TK, Dehdashti F, Wick MR, Monsees BS: Invasive breast cancer: mammographic measurement. Radiology 199: 819-823, 1996

12. Davis PL, Staiger MJ, Harris KB, Ganott MA, Klementaviciene J, McCarty KS Jr., Tobon H: Breast cancer measurements with magnetic resonance imaging, ultrasonography, and mammography. Breast Cancer Res Treatment 37: 1-9, 1996

13. Herrada J, Iyer RB, Atkinson EN, Sneige N, Buzdar AU, Hortobagyi GN: Relative value of physical examination, mammography, and breast sonography in evaluating the size of the primary tumor and regional lymph node metastases in women receiving neoadjuvant chemotherapy for locally advanced breast carcinoma. Clin Cancer Res 3: 1565-1569, 1997

14. Meden H, Neues KP, Roben-Kampken S, Kuhn W: A clinical, mammographic, sonographic and histologic evaluation of breast cancer. Int J Gynaecol Obstet 48: 193-199, 1995

15. Pain JA, Ebbs SR, Hern RP, Lowe S, Bradbeer JW: Assessment of breast cancer size: a comparison of methods. Eur J Surg Oncol 18: 44-8, 1992

16. Tsuboi N, Inomata T, Ogawa Y, Yoshida D, Yoshida S, Moriki T, Kumon M: Changes in the findings of dynamic MRI by preoperative CAF chemotherapy for patients with breast cancer of stage II and III: pathologic correlation. Oncol Rep 6: 727-732, 1999

17. Pierce L, Adler D, Helvie M, Lichter A, Merajver S: The use of mammography in breast preservation in locally advanced breast cancer. Int J Radiat Oncol Biol Phys 34: 571-577, 1996

18. Tohnosu N, Okuyama K, Koide Y, Kikuchi T, Awano T, Matsubara H, Sano T, Nakaichi H, Funami Y, Matsushita K: A comparison between ultrasonography and mammography, computed tomography and digital subtraction angiography for the detection of breast cancers. Surg Today 23: 704-710, 1993

19. Yang WT, Lam WW, Cheung H, Suen M, King WW, Metreweli $\mathrm{C}$ : Sonographic, magnetic resonance imaging, and mammographic assessments of preoperative size of breast cancer. J Ultrasound Med 16: 791-797, 1997

20. Abraham DC, Jones RC, Jones SE, Cheek JH, Peters GN, Knox SM, Grant MD, Hampe DW, Savino DA, Harms SE: Evaluation of neoadjuvant chemotherapeutic response of locally advanced breast cancer by magnetic resonance imaging. Cancer 78: 91-100, 1996

21. Lagalla R, Caruso G, Finazzo M: Monitoring treatment response with color and power Doppler. Eur J Radiol 27(Suppl 2), S149S156, 1998

22. Rieber A, Zeitler H, Rosenthal H, Gorich J, Kreienberg R, Brambs HJ, Tomczak R: MRI of breast cancer: influence of chemotherapy on sensitivity. Brit J Radiol 70: 452-458, 1997

23. Trecate G, Ceglia E, Stabile F, Tesoro-Tess JD, Mariani G, Zambetti M, Musumeci R: Locally advanced breast cancer treated with primary chemotherapy: comparison between magnetic resonance imaging and pathologic evaluation of residual disease. Tumori 85: 220-228, 1999

24. Cheung YC, Chen SC, Su MY, See LC, Hsueh S, Chang HK, Lin $\mathrm{YC}$, Tsai CS: Monitoring the size and response of locally advanced breast cancers to neoadjuvant chemotherapy (weekly paclitaxel and epirubicin) with serial enhanced MRI. Breast Cancer Res Treatment 78: 51-58, 2003

25. Wasser K, Klein SK: Evaluation of neoadjuvant chemotherapeutic response of breast cancer using dynamic MRI with high temporal resolution. Eur Radiol 13: 80-87, 2003

26. Londero V, Bazzocchi M, Del Frate C, Puglisi F, Di Loreto C, Francescutti G, Zuiani C: Locally advanced breast cancer: comparison of mammography, sonography, and MR imaging in evaluation of residual disease in women receiving neoadjuvant chemotherapy. Eur Radiol 14: 1371-1379, 2004 
27. Esserman L, Hylton N, Yassa L: Utility of magnetic resonance imaging in the management of breast cancer: evidence for improved preoperative staging. J Clin Oncol 17: 110-119, 1999

28. Abraham DCJ, Jones RC, Jones SE: Evaluation of neoadjuvant chemotherapeutic response of locally advanced breast cancer by magnetic resonance imaging. Cancer 78: 91-100, 1996

29. LeCarpentier GLT, Fowlkes JB, PB, Roubidoux MA, Moskalik AP Carson PL: Utility of 3D ultrasound in the discrimination and detection of breast cancer. RSNA EJ, 1999

30. Bhatti P, LeCarpentier G, Roubidoux M, Fowlkes J, Helvie M, Carson P: Discrimination of sonographically detected breast masses using frequency shift color Doppler imaging in combination with age and gray scale criteria. J Ultrasound Med 20: 343 350,2001

31. von Minckwitz G, Costa SD, Eiermann W, Blohmer JU, Tulusan AH, Jackisch C, Kaufmann M: Maximized reduction of primary breast tumor size using preoperative chemotherapy with doxorubicin and docetaxel. J Clin Oncol 17: 1999-2005, 1999

32. Minckwitz Gvon, Costa SD, Raab G, Blohmer JU, Eidtmann H, Hilfrich J, Merkle E, Jackisch C, Gademann G, Tulusan AH, Eiermann W, Graf E, Kaufmann MGerman Preoperative A-D, German Adjuvant Breast Cancer Study G. Dose-dense doxorubicin, docetaxel, and granulocyte colony-stimulating factor support with or without tamoxifen as preoperative therapy in patients with operable carcinoma of the breast: a randomized, controlled, open phase IIb study. J Clin Oncol 19: 3506-3515, 2001

33. Tsuboi N, Ogawa $\mathrm{Y}$, Inomata $\mathrm{T}$, Yoshida D, Yoshida S Moriki T, Kumon M: Changes in the findings of dynamic MRI by preoperative CAF chemotherapy for patients with breast cancer of stage II and III: pathologic correlation. Oncol Rep 6: 727-732, 1999

34. Valero V: Primary chemotherapy with docetaxel for the management of breast cancer. Oncology (Huntington) 16: 35-43, 2002

35. Buzdar AU, Hunt KK, Smith TL, Francis D, Ewer M, Booser D, Singletary E, Buchholz T, Sahin A, Hortobagyi GN: Significantly higher pathological complete remission rate following neoadjuvant therapy with trastuzumab, paclitaxel, and anthracycline-containing chemotherapy: initial results of a randomized trial in operable breast cancer with Her-2 positive disease (oral presentation). 2004 ASCO Annual Meeting Proceedings (Post-Meeting Edition) 22, Abstract \#520, 2004

36. Ayers M, Symmans WF, Stec J, Damokosh AI, Clark E, Hess K, Lecocke M, Metivier J, Booser D, Ibrahim N, Valero V, Royce M, Arun B, Whitman G, Ross J, Sneige N, Hortobagyi GN, Pusztai L: Gene expression profiles predict complete pathologic response to neoadjuvant paclitaxel and fluorouracil, doxorubicin, and cyclophosphamide chemotherapy in breast cancer. J Clin Oncol 22: 2284-2293, 2004

37. Sotiriou C, Powles TJ, Dowsett M, Jazaeri AA, Feldman AL, Assersohn L, Gadisetti C, Libutti SK, Liu ET: Gene expression profiles derived from fine needle aspiration correlate with response to systemic chemotherapy in breast cancer. Breast Cancer Res 4: 2002

Address for offprints and correspondence: Anne F. Schott, MD, $1500 \mathrm{E}$. Medical Center Drive, C354 MIB, Ann Arbor, MI 48109-0848; Tel.: +1-734-615-4762; Fax: +1-734-647-8792;E-mail: aschott@ umich.edu 